\title{
Precision Measurement of Displacement with Two Quasi-Orthogonal Signals for Linear Diffraction Grating Interferometric Sensors
}

\author{
Jianglin $\mathrm{BI}^{1, \text { a }}$, Shoubin $\mathrm{LIU}^{2, \mathrm{~b}^{*}}$ \\ ${ }^{1,2}$ Department of Mechanical Engineering and Automation, Harbin Institute of Technology Shenzhen \\ Graduate School, Shenzhen, 518055, China \\ aemail: zxanyy@126.com, bemail: mesbliu@hitsz.edu.cn \\ ${ }^{*}$ Corresponding author
}

Keywords: Displacement Sensor; Diffraction Grating Interferometry; Signal Subdivision; Phase Difference Measurement

\begin{abstract}
Linear diffraction grating interferometer measures displacement via two orthogonal signals generated from the optical outputs of fringes. Due to noises and regulation errors, two signals are not perfect. This paper presents a precision measurement method of displacement for given two non-orthogonal signals. The phase difference of two signals is first calculated by solving an equation derived from correlation principle. The subdivision value is then obtained with a compensation formula. By further counting the cycle number of periodic signal with an amplitude filtering algorithm, accurate value of displacement is outputted. Experimental results show that the proposed method can measure the displacement expressed with two non-orthogonal signals more reliably and accurately.
\end{abstract}

\section{Introduction}

Displacement is one of the most basic geometric parameters, accurate measurement of displacement is of great significance in various fields of industry. Compared to many micro/nano displacement measurement techniques, optical interferometry exhibits advantages of high precision and high resolution. There are two kinds optical interferometric techniques, namely laser interferometry and grating interferometry [1,2]. Figure 1 shows a linear diffraction grating interferometer for displacement measurement. A laser diode emits a laser beam onto a diffraction grating. After reflecting by mirrors, the resulting $\pm 1^{\text {st }}$ order diffracted beams meet together in the plane of photodetectors. Two electrical signals are generated from the optical outputs of interference fringes. After signal conditioning, two signals are sampled synchronously in a DSP sampler. A computer reads the waveforms of two signals and interprets the micro/nano displacement.

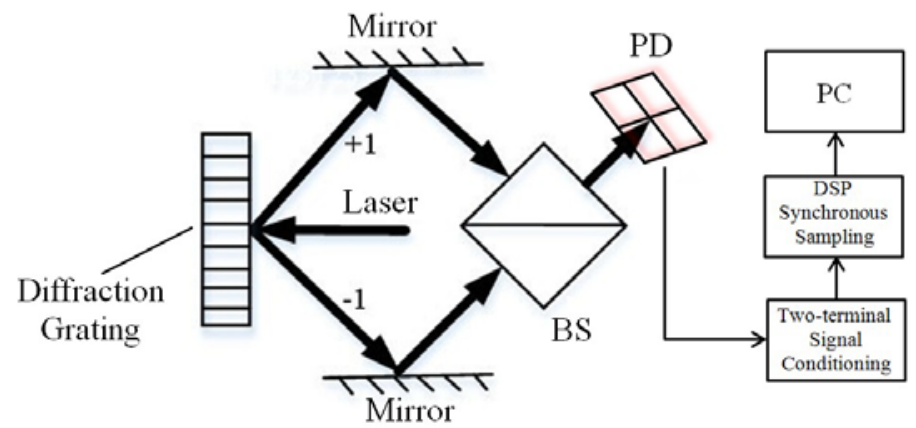

Fig. 1. A linear diffraction grating interferometer for displacement measurement

In a typical grating interferometric displacement measurement system, two signals are well regulated for achieving sine waveforms and a quarter phase difference $(\pi / 2)$. Such two signals are named as two orthogonal signals, or A-quarter-B (i.e., AQB) signals. Once AQB signals are obtained, a subdivision system can provide displacement with subdivision resolution. Unfortunately, due to optical interferences, electrical noises and mechanical regulation errors, two signals are not perfect both in waveform and in phase difference. Such two non-orthogonal signals will generate a 
big subdivision error. A software measuring method of two non-orthogonal signals is desired.

\section{Phase Difference Measurement Based on Correlation Principle}

Currently, there are many methods used in measuring the phase difference of two signals. These methods include cross-zero, correlation algorithm, DFT [3-6] etc. Correlation method calculates phase difference by solving the value of cross-correlation function at a zero-delay of two sine signals with a same frequency [7]. Compared to other methods, correlation method has a good ability of noise suppression. But traditional correlation method exhibits lower precision in the condition of non integer period sampling. An algorithm is thus proposed in this paper to overcome such limitation. The modified correlation algorithm can improve the measuring accuracy of phase difference, and its principle is illustrated as follows.

Two signals with a same frequency can be expressed as

$$
\begin{aligned}
& x(t)=A \cos (\omega t+\theta)+N_{1}(t) \\
& y(t)=B \cos (\omega t+\theta+\varphi)+N_{2}(t)
\end{aligned}
$$

where $A, B$ denote as the amplitudes of the two signals, and $\theta$ is the initial phase of signal $x(t), \theta+\varphi$ is the initial phase of $y(t)$, and $N_{1}(t)$ and $N_{2}(t)$ are noises superimposed on two signals.

Correlation functions of two signals can be calculated by $N$ sampling points with

$$
\begin{aligned}
& R_{x x}(0)=\frac{1}{2}+\frac{1}{2 N} \sum_{n=0}^{N-1} \cos (2 \omega n+2 \theta) \\
& R_{y y}(0)=\frac{1}{2}+\frac{1}{2 N} \sum_{n=0}^{N-1} \cos (2 \omega n+2 \theta+2 \varphi) \\
& R_{x y}(0)=\frac{1}{2}+\frac{1}{2 N} \sum_{n=0}^{N-1} \cos (2 \omega n+2 \theta+\varphi)
\end{aligned}
$$

By adding formula (3) and formula (4), and using some properties of trigonometric function, formula (5) can be written as

$$
\cos ^{2} \varphi-2 \cos \varphi \bullet R_{x y}(0)+R_{x x}(0)+R_{y y}(0)-1=0
$$

By equation (6), phase difference $\varphi$ can be solved as

$$
\varphi=\arccos \left(R_{x y} \pm \sqrt{4 R_{x y}^{2}-4\left(R_{x x}+R_{y y}-1\right)} / 2\right)
$$

Considering the fact of

$$
\frac{A B}{2} \cos \varphi \gg \frac{A B}{2 \omega(k T+\Delta T)} \cos (\omega \Delta T+2 \theta+\omega) \sin (\omega \Delta T)
$$

it is possible to determine the range of phase difference by $R_{x y}(0)$. When $R_{x y}(0)>0$, the range of phase difference is $[0, \pi / 2]$, the phase difference should be calculated as

$$
\varphi=\arccos \left(R_{x y}+\sqrt{4 R_{x y}^{2}-4\left(R_{x x}+R_{y y}-1\right)} / 2\right)
$$

When $R_{x y}(0)<0$, the range of phase difference is $[\pi / 2, \pi]$, the phase difference should be calculated as

$$
\varphi=\arccos \left(R_{x y}-\sqrt{4 R_{x y}^{2}-4\left(R_{x x}+R_{y y}-1\right)} / 2\right)
$$

Thus, the procedure of calculating phase difference with the modified correlation algorithm is

1) Normalizing the amplitudes of two signals;

2) Filtering signals with an adaptive filter;

3) Calculating correlation functions $R_{x x}(0), R_{y y}(0), R_{x y}(0)$ by $N$ sampling points;

4) Calculating phase difference $\varphi$ by formula (9) or formula (10). 


\section{Signal Subdivision}

Typically, the output signals of a displacement are not integer number of cycles. There is a need to calculate the non-periodic part of two signals. The non-periodic part is usually expressed by a phase angle $\alpha$ in a Lissajous figure. In the condition that two signals are orthogonal and have same amplitude, the phase angle $\alpha$ of the non-periodic part can be calculated with a Lissajous circle as [8]

$$
\alpha=\arctan 2\left(I_{\mathrm{y}}, I_{x}\right)
$$

In the condition that two signals have same amplitude but are not in quadrature, the phase angle $\alpha$ of the non-periodic part can be calculated with a Lissajous ellipse as $[9,10]$

$\alpha=\arctan 2\left(I_{y} / I_{x}-\sin \psi, \cos \psi\right)$

where $I_{x}$ and $I_{y}$ are the levels of two signals at the subdivision position, $\psi=\varphi-\pi / 2$ and $\varphi$ is phase difference estimated by the modified correlation algorithm. When $\varphi=\pi / 2$, i.e. two signals are orthogonal, formula (12) becomes formula (11).

Formula (12) is basically a compensation formula of phase angle, and $\alpha$ is a compensated phase angle. Once the phase angle $\alpha$ is obtained, the subdivision displacement can be calculated as

$$
\Delta L=(\alpha / 2 \pi) * l
$$

where $l$ is a half pitch of the diffraction grating.

\section{Counting Cycle Number with an Amplitude Filter}

Because there are distortions in two filtered signals, an amplitude filter is designed for accurate counting the cycle number of two signals. The amplitude filter first calculates the slope of two adjacent sampling points, and the sign of slope is used for indentifying the peaks and valleys in a signal. When the sign of slope changes from positive into negative, the current point is marked as a peak. When the sign of slope changes from negative to positive, the current point is marked as a valley.

The amplitude filter takes a couple of peak and valley. If peak occurs first, the peak is marked as a valid peak. If their height difference is less than a half of the amplitude of a signal, cycle counter does not work. Taking a next couple of peak and valley, if the height difference of the first peak and the last valley is greater than a half of the amplitude, cycle counter works to add 1 . Taking the next couple of peak and valley, the peak is marked as a valid peak again, and doing above process iteratively. Finally, accumulated number of cycle counter subtracts 1 to represent the number of segments of periodic part of a signal.

If valley occurs first, the valley is marked as a valid valley. If the height difference of peak and valley is less than a half of the amplitude of a signal, cycle counter does not work. Taking a next couple of valley and peak, if the height difference of the first valley and the last peak is greater than a half of the amplitude, cycle counter works to add 1 . Taking the next couple of valley and peak, the valley is marked as a valid peak again, and doing above process iteratively. Finally, accumulated number of cycle counter subtracts 1 to represent the number of segments of periodic part of a signal.

Once the cycle number $n$ of integer periods of a signal is obtained, the displacement of integer periods will be $n * l$. The total displacement measured will be

$$
L=n * l+\Delta L_{1}+\Delta L_{2}
$$

where $l$ is a half pitch of the diffraction grating, $n$ is the cycle number of integer periods, $\Delta L_{1}$ and $\Delta L_{2}$ are two subdivision displacements at starting and ending positions calculate by formula (13). 


\section{Experimental Results}

To verify the effectiveness of estimated phase difference with the modified correlation algorithm, two simulated sine signals are generated. Two signals have same amplitude and a same angular frequency ( $\omega=\pi / 50$ ). Their initial phases are $\theta=0^{\circ}$ and $\theta+\varphi=95^{\circ}$, and phase difference is $95^{\circ}$. The two signals are shown in Figure.2, and the calculating results of phase difference varied with $N$ sampling points are shown in Table 1.

As can be seen from Table 1, the phase difference is not affected by the number of sampling points. The modified correlation algorithm is of high accuracy. In addition, the error of phase difference is smaller when the number of sampling points $N$ is around 200. So the number of sampling points is chosen as $N=200$. Comparing to other traditional correlation algorithms, the proposed algorithm overcomes the limitation that the number of sampling points must be an integer number of periods.

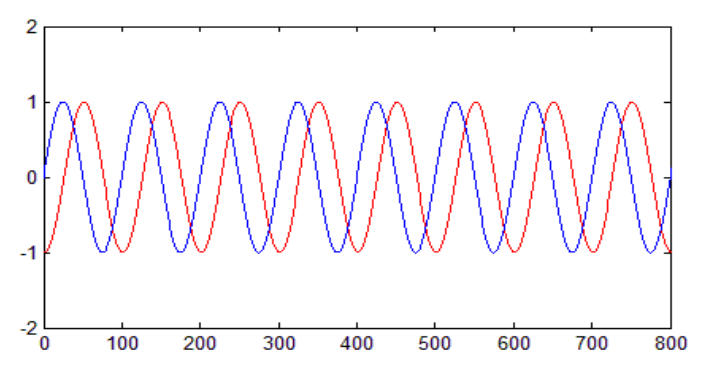

Fig.2 Two sine signals with a phase difference of $95^{\circ}$
Table 1 The error of phase difference with $N$

\begin{tabular}{ccc}
\hline$N$ & Phase difference $\left(^{\circ}\right)$ & Error $\left(^{\circ}\right)$ \\
\hline 100 & 96.006 & 1.006 \\
150 & 95.708 & 0.708 \\
200 & 95.423 & 0.423 \\
250 & 95.470 & 0.470 \\
300 & 95.471 & 0.471 \\
\hline
\end{tabular}

To verify the proposed precision measuring method of displacement, a measuring experiment is conducted. In the grating interferometric displacement measurement system as illustrated in Figure 1 , the diffraction grating is driven by a precision piezoelectric stage only in one direction. The piezoelectric stage (SFS-H60XY by Sigma koki) has a step resolution of $1 \mathrm{~nm}$. A known displacement $L=20.033 \mu \mathrm{m}$ is applied. The diffraction grating has a line density of $2400 \mathrm{l} / \mathrm{mm}$ (half pitch of grating $=0.2083 \mu \mathrm{m}$ ). The sampling frequency is $1 \mathrm{kHz}$. Two sampled signals before filtering and after filtering are shown in Figure 3.

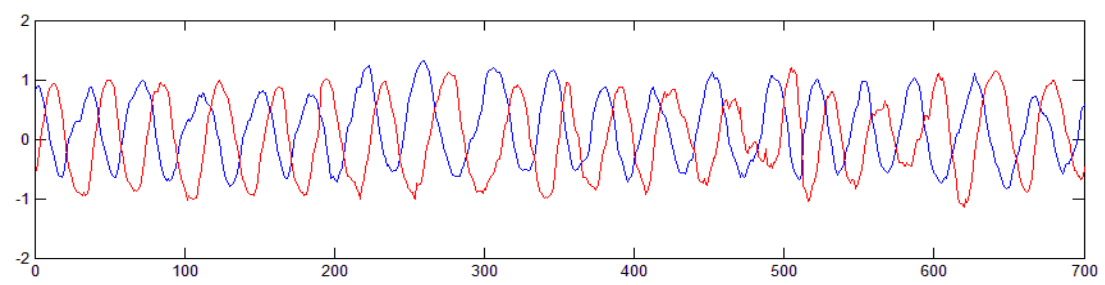

(a)

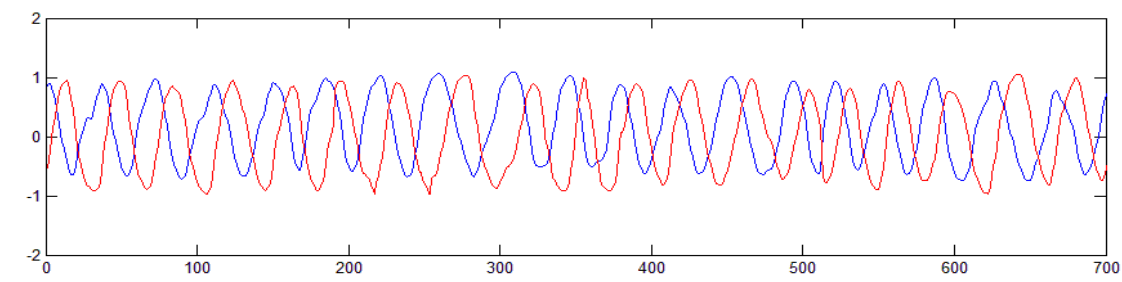

(b)

Fig. 3. Partial waveforms of two measured signals resulted by a displacement:

(a) two original signals and (b) two filtered signals

Table 2 The measuring result and error of the displacement

\begin{tabular}{cccc}
\hline $\begin{array}{c}\text { True displacement } \\
(\mu \mathrm{m})\end{array}$ & $\begin{array}{c}\text { Phase difference } \\
\left.{ }^{\circ}\right)\end{array}$ & $\begin{array}{c}\text { Measured displacement } \\
(\mu \mathrm{m})\end{array}$ & $\begin{array}{c}\text { Error } \\
(\mu \mathrm{m})\end{array}$ \\
\hline
\end{tabular}


By using the modified correlation algorithm, the phase difference of two signals after filtering is calculated as $121.079^{\circ}$, the number of cycle counter is $n=96$, the total displacement measured is $20.067 \mu \mathrm{m}$, and measuring error is $0.034 \mu \mathrm{m}$. The displacement resolution of the grating interferometric displacement measurement system is less than $10 \mathrm{~nm}$. The experimental results are shown in Table 2.

\section{Conclusion}

This paper presents a precision measurement method of displacement for given two non-orthogonal signals outputted from a diffraction grating interferometric displacement measurement system. The phase difference of two signals is calculated by a modified correlation algorithm. The subdivision displacement is calculated with a compensated phase angle. Cycle number of periodic part of a signal is counted with an amplitude filter algorithm. A measuring experiment of displacement is performed and experimental results show that the proposed method is feasible and of a good ability of noise suppression.

\section{Acknowledgement}

This research was sponsored by the Shenzhen Basic Research Project grant No. JCYJ20140417172417154.

\section{References}

[1] N. Yim, M. EOM and S. Kim: Meas. Sci. Technol. Vol. 11(2000), p. 1131

[2] A. M. Dobosz: Optical Engineering Vol. 33 (1994), p. 897

[3] H. So: IEE Proceedings-Radar, Sonar and Navigation Vol.148 (2001), p. 318

[4] H. So: IEEE Trans. IM. Vol.54 (2005), p. 2501

[5] Y. Jenq, P. Crosby: IEEE Trans. IM. Vol. 37 (1988), p. 529

[6] C. Vennila, G. Laksjminarayanan and S. Ko: Circuits Systems \& Signal Processing Vol. 31 (2012), p. 1049

[7] D. Hertz: Signal Processing Vol. 8 (1985), p. 235

[8] M. Dobosz, G. Zamiela: Optics \& Laser Technology Vol. 44 (2012), p. 1620

[9] C. Wu, C. Su, G. Peng: Meas. Sci. Technol. Vol. 7 (1996), p. 520

[10]K. Birch: Precision Engineering Vol. 12 (1990), p. 195 